\title{
Puesta al día en manejo de la obstrucción nasal por colapso valvular
}

\section{Update in nasal obstruccion management caused by valvular collapse}

\author{
Alfredo Naser G1, Patricio Tabilo C¹, Gustavo Bravo C¹, María I Carrasco D².
}

\begin{abstract}
RESUMEN
La estenosis de la válvula nasal aparece como una complicación frecuente de rinoplastía o traumatismo nasal. Existen diferentes técnicas para corregir el colapso de la válvula nasal; generalmente se utilizan injertos de tejido, que en algunas ocasiones son insuficientes para resolver la alteración y mejorar la función respiratoria. La válvula nasal funciona en base a la resistencia de la ley Starling, que consiste en un tubo semirrígido con un segmento flexible; cuando la presión inspirada excede un nivel crítico, éste se colapsa y provoca la alteración en su fisiología; por tanto, es necesario cierto grado de rigidez en el componente lateral nasal para evitar el colapso durante la inspiración. Cuando se ve afectado su funcionamiento causa frecuentemente obstrucción nasal.
\end{abstract}

Palabras clave: Colapso de válvula nasal, obstrucción nasal, rinoplastía.

\begin{abstract}
Nasal valve stenosis commonly presents as a postoperative complication of rhinoplasty or facial trauma. Various techniques have been reported to correct nasal valve stenosis through the use of methods that support the nasal valve with cartilage, rib graft, although these tissues are not useful in some cases to improve the nasal breathing. The nasal valve functions as the law Starling resistor, which is a semirigid tube with a flexible segment, when the inspired partial pressure exceeds a critical level, it collapses and causes nasal obstruction, and therefore need some degree of rigidity in nasal lateral component to avoid collapse during inspiration. When the nasal valve is affected frequently causes nasal obstruction.
\end{abstract}

Key words: Nasal valve collapse, nasal obstruction, rhinoplasty.

La rinoplastía es una de las cirugías estéticas más realizadas en el mundo y entre las complicaciones más frecuentes de ésta se encuentra el colapso valvular generalmente por una excesiva resección del cartílago alar.

La válvula nasal está delimitada por el ángulo formado entre el borde inferior del cartílago trian- gular y el tabique nasal. Dicho ángulo tiene un valor de $10^{\circ}-15^{\circ}$ y representa la región nasal con menor área de sección de corte ${ }^{1}\left(55-83 \mathrm{~mm}^{2}\right)$; cuando ese ángulo es menor de este valor se considera que la válvula nasal está colapsada, lo cual tiene lugar cuando no es capaz de soportar la presión inspiratoria negativa.

Médico Otorrinolaringólogo, Hospital Clínico Universidad de Chile.

2 Interna Medicina, Facultad de Medicina, Universidad de Chile. 
En 1903 Mink fue el primero en describir la significancia anatómica de la válvula nasal y Bridger en el año 1970 fijó los límites que están dado por: cartílago lateral, septum, cabeza del cornete inferior, piso de la fosa nasal y por lateral apertura piriforme².

La obstrucción nasal debido a anormalidades de la válvula nasal puede ser debido a problemas dinámicos 0 estéticos y es una de las más importantes razones de obstrucción nasal (Tabla 1).

Elwany y Thabet reportaron que el $13 \%$ de los pacientes que tienen obstrucción nasal es debido a disfunción de la válvula nasal ${ }^{3}$.

Con el gran advenimiento que hay hoy en día de la cirugía estética nasal se ven cada día más disfunciones de la válvula nasal dada principalmente por el excesivo retiro de cartílago alar, lo que produce el colapso de esta válvula. De hecho se ha visto que luego de una rinoplastía el área de sección de corte de la válvula nasal se reduce en $22 \%$ a $25 \%{ }^{1}$ por lo que en algunos pacientes basta con eso para que se produzcan los problemas de obstrucción nasal dado por el colapso valvular.
Hay múltiples técnicas para mejorar el colapso valvular nasal, ninguna de ellas 100\% efectivas, probablemente porque la etiología es multifactorial y hay factores individuales que varían en cada paciente ${ }^{5}$, centrándose la mayor parte de ellas en la propia válvula nasal o el cartílago triangular, es decir generalmente en un solo factor y no en la etiología multifactorial de ésta.

Esta revisión tiene como objetivo mostrar las técnicas que se ocupan actualmente para mejorar el colapso valvular, ninguna de ellas $100 \%$ efectivas pero con buenos resultados.

Dentro de las técnicas destacan:

\section{CARTÍLAGOS EXPANSORES ${ }^{6}$}

0 más bien conocidos como Spreader Graft son listones de 1-2 $\mathrm{mm}$ de ancho y de al menos $15 \mathrm{~mm}$ de largo que son obtenidos del cartílago septal y son puestos submucopericóndricamente entre el septum y el cartílago alar superior y fijados con catgut crómico 5/0 (Figura 1). La función de estos cartílagos

Tabla 1. Clasificación patológica de la obstrucción de válvula nasal ${ }^{4}$

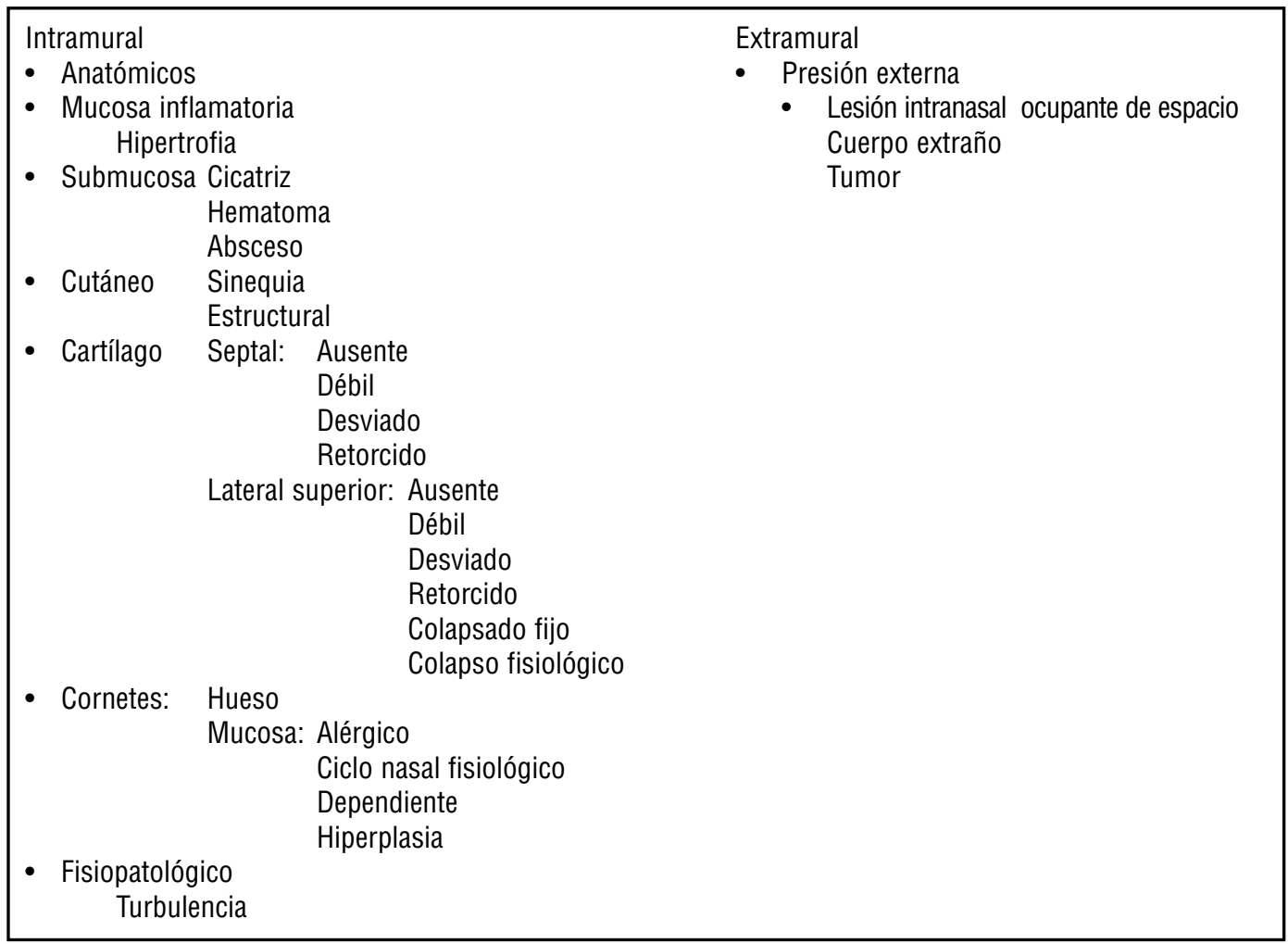


expansores es mejorar el flujo en el área de la válvula nasal. Son diseñados para lateralizar el cartílago lateral superior, incrementando el área de la válvula nasal por lo que deberían ser usados cuando el septum nasal es débil y necesita refuerzo.

\section{CARTÍLAGO EN LISTON ${ }^{7}$}

Se ocupa principalmente en rinoplastías funcionales para ampliar y fortalecer la pared nasal lateral supraalar pero podría tener el inconveniente de aportar más carga en una zona en teoría más débil por lo que podría exacerbar el problema ${ }^{8}$.

El injerto puede ser puesto por vía endonasal 0 vía abierta, de cualquiera de las dos formas éste debe ser puesto dentro de un bolsillo en el punto de máximo colapso de la pared nasal lateral usando cartílago septal 0 de la concha auricular. Este bolsillo va desde la apertura piriforme a la unión del tercio lateral y medial de la crura lateral. En la mayoría de los casos el injerto mide $10-15 \mathrm{~mm}$ de

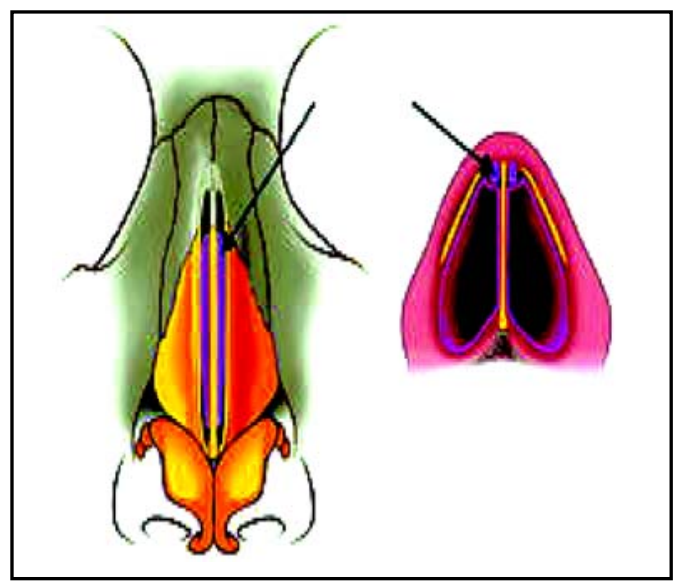

Figura 1. Tomado de Acta Otolaryngol 2000; 120: 312-5. largo y 4-8 mm de ancho. En esta serie de 46 pacientes $^{12} 98 \%$ notó mejoría en sus síntomas obstructivos en un promedio de seguimiento de 5 años (Figuras 2, 3 y 4).

\section{CARTÍLAGO EN MARIPOSA ${ }^{4}$}

Esta técnica es principalmente usada en pacientes que tienen obstrucción nasal secundaria a rinoplastía. El injerto de mariposa se obtiene de la concha auricular izquierda por la facilidad del cirujano de estar de pie al lado derecho. La incisión en la concha auricular es hecha 1-2 mm dentro del borde libre del antihelix y se extiende hacia la cruz inferior, $1 \mathrm{~mm}$ bajo el helix superiormente, inferiormente la incisión se extiende 1$2 \mathrm{~mm}$ dentro del antitrago. La porción ascendente de cartílago de la concha auricular u hombro, es el sitio más óptimo para el injerto en mariposa porque tiene una curvatura sin mayores problemas. La colocación del injerto en alas de mariposa es hecha a través de técnica abierta o cerrada indistintamente dependiendo

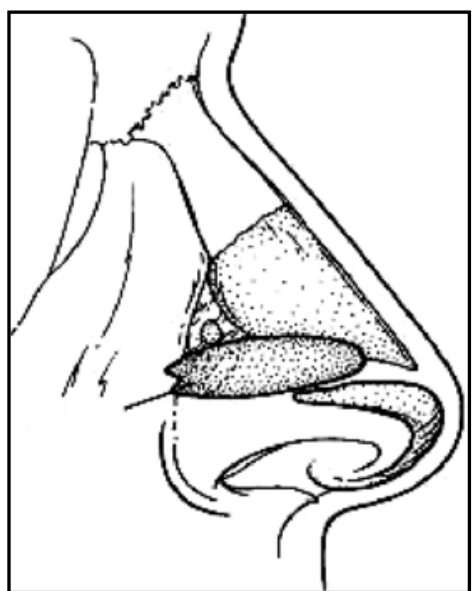

Figura 2. Tomada de Laryngoscope 2005; 115: 903-9.

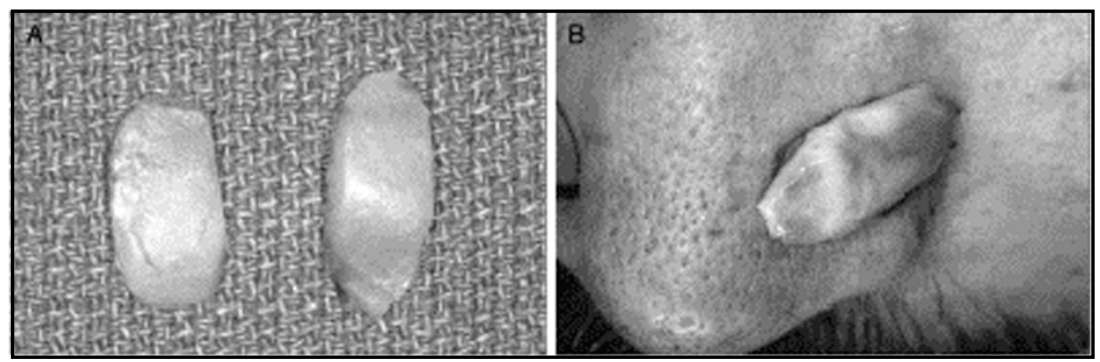

Figura 3. Tomada de Laryngoscope 2005; 115: 903-9. 


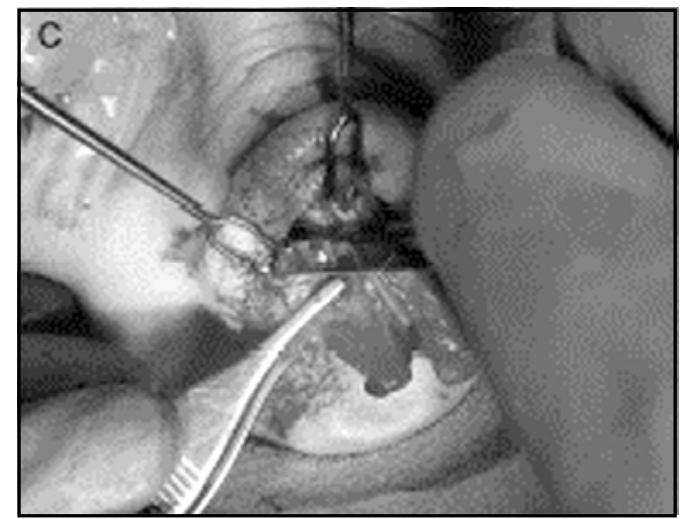

Figura 4. Tomada de Laryngoscope 2005; 115: 903-9.

de la habilidad del cirujano. En caso de un abordaje cerrado (con una incisión intercartilaginosa unido medialmente con una incisión transfixiante superior completa, la piel/SMAS es elevado del rinion cefálicamente y lateralmente hasta el cartílago lateral superior). El injerto es puesto a través de la incisión con la forma de $\mathrm{V}$ apuntando caudalmente y puesto simétricamente sobre el dorso nasal. El injerto es fijado con sutura 5/0. La sutura es puesta de profundo a superficial a través del borde caudal del cartílago lateral superior (Figura 5). En el caso de esta técnica los autores describen $97 \%$ de resolución del problema obstructivo de los pacientes y sólo $3 \%$ de no resolución de su problema obstructivo, distinto es el caso en aquellos pacientes que también requerían alguna corrección estética en donde el 86\% quedó conforme con su nariz, $11 \%$ no varió su nariz y $3 \%$ dijo que su nariz quedó peor de como la tenía. Stucker y Hoasjoe ${ }^{11}$ también reportaron $100 \%$ de mejoría en 5 pacientes sometidos a esta técnica.

\section{INJERTO EN ALAS DE GAVIOTA9}

Esta técnica creada por Pedroza en 1981 fue diseñada para reemplazar el cartílago alar inferior y corregir una gran variedad de defectos estéticos nasales. Generalmente basta con un trozo de cartílago obtenido de la concha auricular (A). Luego se divide este trozo de cartílago alar en dos mitades iguales a lo largo de su eje axial (B). Cada mitad es medida para crear un neodomo dejando $1 \mathrm{~cm}$ para cruz medial y $2 \mathrm{~cm}$ para cruz medial (C). Estos 2 neodomos son suturados primero posteriormente 2-3 cm bajo los neodomos y luego anteriormente 6-8 $\mathrm{mm}$ bajo los neodomos (E) (Figura 6). El acceso nasal puede ser por vía externa, incisión marginal y delivery.

Los resultados en pacientes sometidos a esta técnica fue de una mejoría del $100 \%$ de los síntomas obstructivos y el grado de satisfacción con esta técnica fue de $95 \%$.

\section{COLGAJO EN «J»8}

Se basa en la teoría que supone que el colapso valvular es ocasionado por la pérdida del tejido fibroadiposo que fija la cruz lateral del cartílago alar a la apertura piriforme, lo cual provoca el prolapso de la cruz lateral hacia el paso aéreo y el subsiguiente estrechamiento del ángulo de la válvula nasal ${ }^{8}$. Por lo tanto esta técnica permitiría mejorar tanto la obstrucción nasal como los defectos estéticos asociados al colapso nasal. La incisión comienza en la región de la cúpula y se prolonga lateralmente siguiendo el borde caudal y la curvatura de la cruz lateral del cartílago alar; una vez que se alcanza la parte más lateral del cartílago alar la incisión se curva hacia otras obteniendo una incisión con forma de J. Posteriormente se diseca un plano suprapericondrial sobre el cartílago alar. La disección se prolonga hacia la cúpula y superiormente hacia el cartílago triangular y se obtiene un colgajo condrocutáneo de base superomedial del que se tracciona en dirección inferolateral hasta visualizar el punto en que la válvula nasal se abre. A 


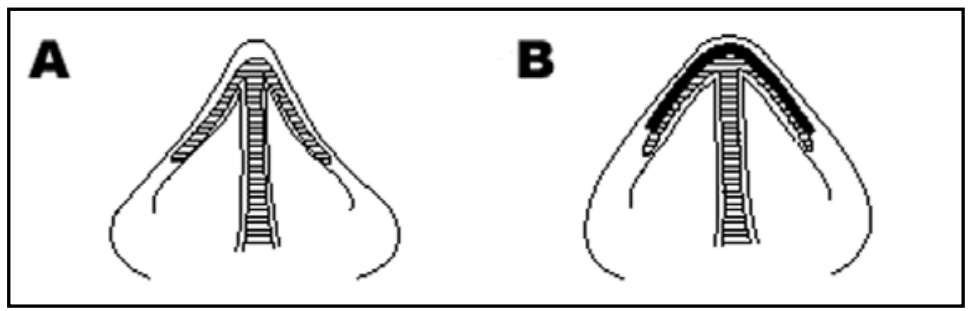

Figura 5. Tomado de Laryngoscope 2002; 112: 1917-25.
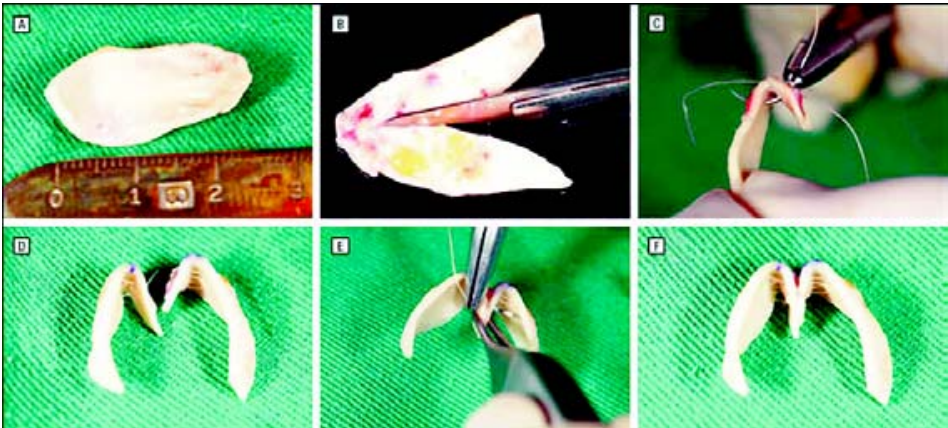

Figura 6. Tomada de Arch Facial Plast Surg 2006; 8(6): 396-403.

continuación se reseca el tejido del colgajo que sobrepasa la incisión realizada en forma de dos fragmentos compuestos de piel y cartílago de los bordes del colgajo. El primero se escinde en forma curvilínea de la parte caudal del colgajo y el segundo procede del borde lateral del colgajo e incluye una parte completa del cartílago alar. La cantidad de tejido

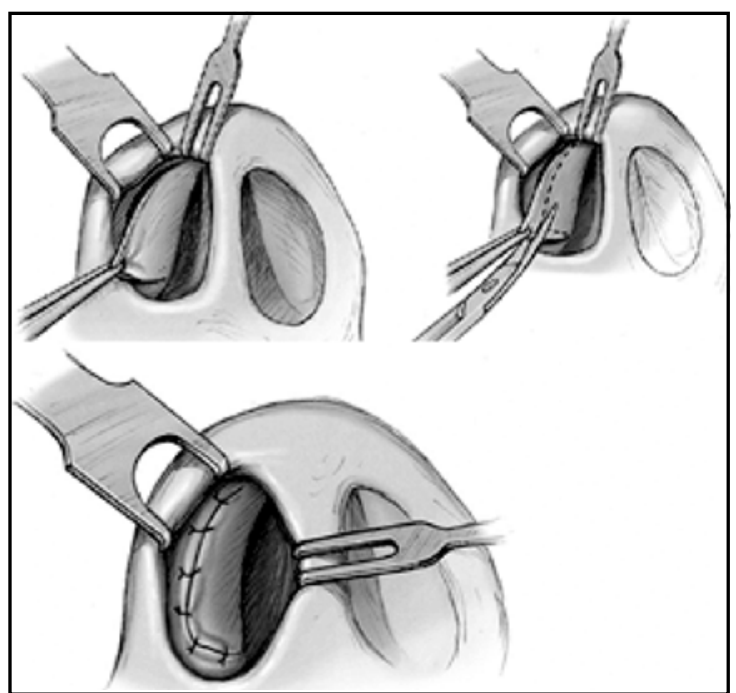

Figura 7. Tomada de Acta Otorrinolaringol Esp 2006; 57: 350-4. recortado es variable y depende de la laxitud de la válvula así como de la tensión que sea necesario aplicar al colgajo para que la válvula se abra pero, en general, se recomienda recortar de $1 \mathrm{a} 2 \mathrm{~mm}$ en el caso del primer fragmento y de 2 a $4 \mathrm{~mm}$ del segundo, si bien se debe evitar resecar demasiado tejido en la obtención del primer fragmento para no dañar el cartílago alar. Se fija el colgajo en posición inferolateral con 2 ó 3 puntos de Monocry ${ }^{\circledR} 3 / 0$ (Figura 7). Todos los pacientes refieren haber mejorado sustancialmente desde la intervención y en el seguimiento promedio de 420 días no hay ninguna recidiva del colapso valvular y solo uno (5\%) quedó disconforme del punto de vista estético.

\section{PUNTO DE LATERALIZACIÓN DEL COLAPSO VALVULAR ${ }^{10}$}

Es una técnica simple de realizar, que se puede realizar en la oficina bajo anestesia local. Se realiza a 3-4 $\mathrm{mm}$ del borde del hueso nasal donde se hace una incisión pequeña, bajo el sistema musculoaponeurótico periostio superficial (SMAS), el cual es elevado unos pocos milímetros 
con un elevador de periostio. Una segunda incisión intercartilaginosa intranasal de $5 \mathrm{~mm}$ es hecha a 10 largo del borde caudal del cartílago lateral superior. Una sutura de Prolene $3 / 0$ es necesaria. Luego la aguja es doblada y acomodada en la frente, la aguja entra en la incisión anterior e inferior al canto medial del ojo bajo la capa SMAS del hueso nasal, enganchando la porción cefálica del cartílago lateral superior y posteriormente entre la mucosa nasal y el cartílago lateral superior para emerger de la incisión intranasal intercartilaginosa. La aguja se retira y se lleva de vuelta a través de la misma incisión intranasal intercartilaginosa pero esta vez lateral al cartílago lateral superior para emerger por el sitio original de entrada bajo el canto medial del ojo. Como el nudo es ligeramente más apretado, el borde caudal del cartílago lateral superior se ve lateralizado. La sutura es cortada lo más posible y el nudo queda debajo de la capa SMAS (Figura 8). Dentro de las ventajas de esta técnica es que el paciente no es sometido a anestesia general y los autores refieren reducción en una escala de obstrucción nasal preoperatorio de 6-9 de 10 a 0-2 de 10 y obviando la septoplastía en los 12 pacientes sometidos a esta técnica en esta serie.

\section{BIBLIOGRAFÍA}

1. Timur Akcman, Oren Friedman, Ted Cook. The Effect on Snoring of structural nasal valve dilatation with a butterfly graft. Arch Otolaryngol Head Neck Surg 2004; 130: 1313-18.

2. MARTYN Golchin. Alar expansion and Reinforcement. Arch Facial Plast Surg 2006; 8: 293-9.

3. Elwany Thabet. Obstruction of the nasal valve. $J$ Laryngol Otol 1996; 110: 221-4.

4. Madison Clark, Ted Cook. The Butterfly Graft in functional secondary rhinoplasty. Laryngoscope 2002; 112: 1917-25.

5. Mendelsonhn Golchin. Alar expansion and reinforcement. Arch Facial Plast Surg 2006; 8: 293-9.

6. Orhan Ozturan. Techniques for the improvement of the internal nasal valve in functional-cosmetic nasal surgery. Acta Otolaryngo/2000; 120: 312-5.

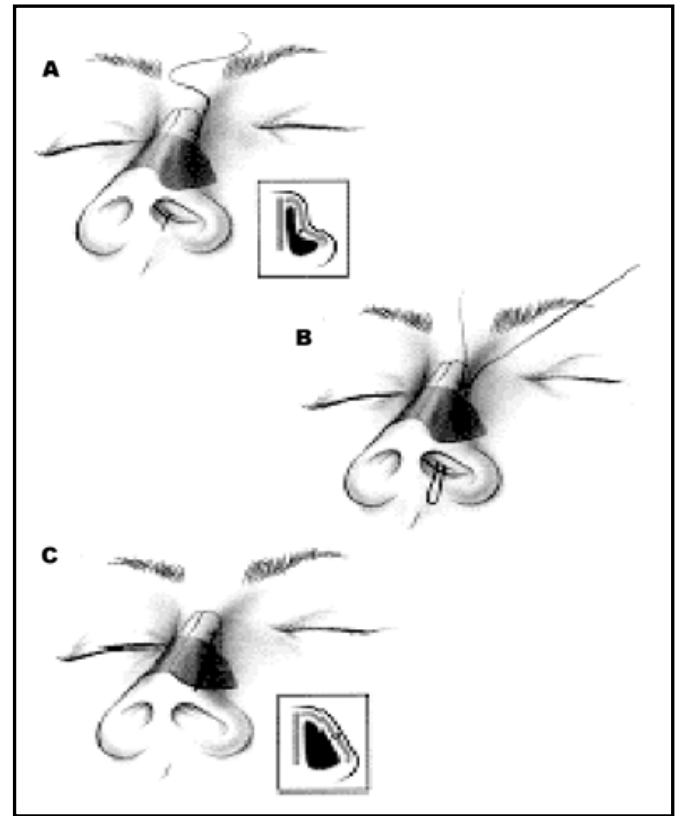

Figura 8. Tomada de Laryngoscope 2003; 113: 2052-4.

7. Egan Kim. A Novel intranasal stent for functional rhinoplasty and nostril stenosis. Laryngoscope 2005; 115: 903-9.

8. GutiérRez, Tapia, Albalad. El colgajo en «J» de la cruz lateral del cartílago alar en el tratamiento del colapso de la válvula nasal. Acta Otorrinolaringol Esp 2006; 57: 350-4.

9. Pedroza F, Anjos GC, Patrocinio LG. Seagull wing graf: a technique for the replacement of lower lateral cartilages. Arch Facial Plast Surg 2006; 8(6): 396-403.

10. Syed Gauthier. How I do It: lateralizing the collapsed nasal valve. Laryngoscope 2003; 113: 2052-4.

11. Stucker FJ, HoAsjoe. Nasal reconstruction with conchal cartilage: correcting valve and lateral nasal collapse. Arch Otolaryngol Head Neck Surg 1994; 120: 653-8.

12. TORIUMI DM, Josen J. Use of alar batten grafts for correction of nasal valve collapse. Arch Otolaryngol Head Neck Surg 1997; 123: 802-8.

\footnotetext{
Dirección: Dr. Alfredo Naser González

Santos Dumont 999. Independencia. Santiago de Chile Fax: 7378088

E mail: aanaserg@gmail.com
} 